\title{
Interleukin-1: The Pros and Cons of Its Clinical Relevance
}

\author{
*S. Shaldon, †C. Dinarello, ‡F. Port, and §M. Kluger
}

${ }^{*}$ Department of Nephrology, University Hospital, Nimes, France, †Department of Medicine, Tufts University School of Medicine, Boston, MA, U.S.A., ‡Department of Internal Medicine, University of Michigan Medical School, Ann Arbor, MI, U.S.A., and §Celus Immune Corporation, Palo Alto, CA, U.S.A.

Dr. Shaldon introduced the panel and then reviewed the background to the launching of the interleukin-1 (IL-1) hypothesis in 1983. He stressed that in 1982 at the Tegernsee dialysis meeting of the German Clinical Society of Nephrology, Drs. Dinarello and Port had discussed mechanisms of fever in dialysis patients with contaminated dialysate. Subsequently, it was demonstrated that endotoxin could pass the dialysis membrane, and that C5a could activate monocyte IL-1 production. Following the cloning of IL-1 in 1984 and tumor necrosis factor (TNF) in 1985, it became obvious that both monokines were endogenous pyrogens and intimately associated with the acute-phase response. Thus, the scene was set to modify the name to the monokine hypothesis.

Dr. Dinarello then reviewed the in vitro and animal effects of recombinant IL-1B and TNF, and suggested that synergism between the two monokines was extremely important in achieving protein catabolism in rats as well as shock in rabbits. He then presented recent data using radioimmunoassay (RIA) methods for measuring these monokines in uremic plasma and confirmed the published bioassay methods that there was a rise in both monokines during dialysis.

Dr. Port reviewed his own work on attempting to demonstrate the induction of IL-1 from monocytes that were recovered from dialyzers after use. He suggested that even though the results were posi-

\footnotetext{
Received January 1988.

Address correspondence to Dr. S. Shaldon at 86 Rue de Grezac, Montpellier 34080, France.

This work was a panel session presented at the VIth World Congress of the International Society for Artificial Organs and the XIVth Congress of the European Society for Artificial Or-
} gans. tive, the degree of activation was so small that it was dubious if they could be responsible for any of the clinical effects seen in long-term dialysis patients.

Dr. Kluger reviewed all the published work of Drs. Dinarello, Shaldon, Bingel, Koch, and Lonnemann relevant to the $\mathrm{IL}-1$ hypothesis and concluded that although the hypothesis was ingenious, the data to date were not sufficiently convincing to support the hypothesis. He started by quoting Thomas Huxley, "The great tragedies of science, the slaying of beautiful hypotheses by ugly facts," and finished with another Huxley quote, "We are prone to see what lies behind their eyes rather than what appears before them."

In the lively discussion that followed, Dr. Shaldon quoted Popper on the unprovableness of any hypothesis and the need for modification in the light of new knowledge. He objected to Dr. Kruger's mathematical analysis of qualitative bioassay data on IL-1 measurements and suggested that RIA methodology validated by bioassay would lead to more precise understanding of the problem. Dr. Dinarello agreed and pointed out that all the bioassay data were measuring IL-6 as well as IL-1 and would need to be repeated. However, preliminary results suggested that TNF and IL-1B levels rose during dialysis when measured by RIA and were significantly higher than normal at their peak values. He affirmed the need to modify the hypothesis and to call it the monokine hypothesis. However, both Dr. Shaldon and Dr. Dinarello were strongly of the opinion that the concept of dialysis activation of human blood monocytes by the membrane, C5a, dialysate endotoxin, and acetate was real and potentially detrimental for long-term dialysis patients. Drs. Port and Kruger remained unconvinced on the present evidence and reserved their judgment. 